\title{
Free transverse vibrations of cracked nanobeams using a nonlocal elasticity model
}

\author{
J. Loya, J. López-Puente, R. Zaera, and J. Fernández-Sáez ${ }^{\text {a) }}$ \\ Department of Continuum Mechanics and Structural Analysis, University Carlos III of Madrid, \\ Avda. de la Universidad 30, Leganés, Madrid 28911, Spain
}

\begin{abstract}
In this paper, flexural vibrations of cracked micro- and nanobeams are studied. The model is based on the theory of nonlocal elasticity applied to Euler-Bernouilli beams. The cracked-beam model is established using a proper modification of the classical cracked-beam theory consisting of dividing the cracked element into two segments connected by a rotational spring located at the cracked section. This model promotes a discontinuity in bending slope, which is proportional to the second derivative of the displacements. Frequency equations of cracked nanobeams with some typical boundary conditions are derived and the natural frequencies for different crack positions, crack lengths, and nonlocal length parameters are calculated. The results are compared with those corresponding to the classical local model, emphasizing the differences occurring when the nonlocal effects are significant.
\end{abstract}

\section{INTRODUCTION}

Many micro- or nanoelectromechanical systems (MEMS or NEMS) devices incorporate structural elements such as beams and plates at a micro- (or nano-) length scale. Size effects are significant in the mechanical behavior of these structures in which dimensions are small and comparable to molecular distances. Since the atomic and molecular models require great computational effort, simplified models are useful for analyzing the mechanical behavior of such devices.

Classical continuum mechanics cannot predict the size effect, due to its scale-free character. Among the sizedependent continuum theories, the theory of nonlocal continuum mechanics initiated by Eringen (1972) and Eringen and Edelen (1972) has been widely used to analyze many problems, such as wave propagation, dislocation, and crack singularities.

From the pioneer work of Peddieson et al. (2003), applying the simplified model proposed by Eringen (1983) for problems involving nanotechnology, several studies have been devoted to different aspects of the mechanical behavior of beams, plates, and shells on the nanoscale. Thus, the nonlocal theory of elasticity has been used to address the static bending behavior, buckling, and free transverse vibrations of microbeams and nanobeams, as well as carbon nanotubes [Sudak (2003), Wang (2005), Xu (2006), Wang et al. (2006), Wang and Varadan (2006), Lu et al. (2006), Wang et al. (2007), Wang and Liew (2007), Lu (2007), Lu et al. (2007), and Heireche et al. (2008)]. Reddy (2007) applied the Eringen nonlocal elastic constitutive relations to derive the equation of motion of various kinds of beam theories available (Euler-Bernoulli, Timoshenko, and Reddy and Levinson) and reached analytical and numerical solutions on static deflections, buckling loads, and natural frequencies. As Reddy (2007) and Lu et al. (2007) have pointed out, some published works [Wang (2005), Wang et al. (2006), Wang and Varadan

${ }^{a)}$ FAX: +349162499 73. Electronic mail: ppfer@ing.uc3m.es.
(2006), and Wang and Liew (2007)] do not adequately consider the nonlocal effect in writing the shear stress-strain relation of the Timoshenko beam theory. It is important to underline that all these works refer to intact devices; although the probability of finding defects is very low, the presence of a defect could dramatically alter the structural behavior of the component. An example of the importance of this kind of analysis is the work of Luque et al. (2006), who have studied the tensile behavior of cylindrical copper wires of nanometric diameter, considering atomically sharp surface cracks. These analyses have been carried out using molecular-dynamics techniques.

In the present work, the flexural vibrations of cracked micro- and nanobeams are studied. In the next section the general constitutive equations of nonlocal elasticity theory are briefly summarized. Section III gives the governing equations of the dynamics of a nonlocal Euler-Bernoulli beam. The cracked beam model is established in Sec. IV, using a proper modification of the classical cracked-beam model by dividing the cracked element into two segments connected by a rotational spring located at the cracked section. This model promotes a discontinuity in bending slope which is proportional to the second-derivative of the displacements. Natural frequencies from cracked nanobeams with some typical boundary conditions are calculated for different crack positions, crack severities, and values of the nonlocal length parameter. The results are compared with those corresponding to the classical local model, highlighting the differences occurring when nonlocal effects are significant.

\section{CONSTITUTIVE RELATIONS FOR THE ERINGEN NONLOCAL ELASTICITY THEORY}

The theory of nonlocal elasticity, developed by Eringen (1972), Eringen (2002), and Eringen and Edelen (1972) states that the nonlocal stress-tensor components $\sigma_{k l}$ at any point $\mathbf{x}$ in a body depend not only on the strain at $\mathbf{x}$ (local theory) but also on strains at all points of the body. For 
homogeneous and isotropic nonlocal elastic solids, the constitutive relations can be expressed in an integral form as

$$
\sigma_{k l}(\mathbf{x})=\int_{\Omega} \alpha\left(\left|\mathbf{x}^{\prime}-\mathbf{x}\right|, \tau\right) t_{k l}(\mathbf{x}) d \Omega\left(\mathbf{x}^{\prime}\right),
$$

where $t_{k l}$ are the components of the classical local stress tensor at point $\mathbf{x}$, which are related to the components of the linear strain tensor $\varepsilon_{k l}$ by the conventional constitutive relations for a Hookean material, i.e.,

$$
t_{k l}(\mathbf{x})=\lambda \varepsilon_{r r}(\mathbf{x}) \delta_{k l}+2 G \varepsilon_{k l}(\mathbf{x}) .
$$

The kernel $\alpha\left(\left|\mathbf{x}^{\prime}-\mathbf{x}\right|, \tau\right)$ is the nonlocal modulus which incorporates into the constitutive relation the nonlocal effect of the stress at point $\mathbf{x}$ produced by local strain at the point $\mathbf{x}^{\prime}$. Here, $\left|\mathbf{x}-\mathbf{x}^{\prime}\right|$ is the Euclidean distance and $\tau$ is given by

$$
\tau=\frac{e_{0} a}{l}
$$

which represents the ratio between a characteristic internal length $a$ (e.g. a $\mathrm{C}-\mathrm{C}$ bond, lattice parameter, and granular distance) and characteristic external one $l$ (e.g., crack length and wavelength). Meanwhile, $e_{0}$ is an adjusting constant, dependent on each material, which needs to be determined from experiments or by matching dispersion curves of plane waves with those of atomic-lattice dynamics.

According to Eringen (1983), for a class of physically admissible kernel $\alpha\left(\left|\mathbf{x}^{\prime}-\mathbf{x}\right|, \tau\right)$ it is possible to represent the integral constitutive relations given by Eq. (1) in an equivalent differential form as

$$
\left[1-\left(e_{0} a\right)^{2} \nabla^{2}\right] \sigma_{k l}=t_{k l} .
$$

Thus, the scale coefficient $e_{0} a$ takes into account the size effect on the response of micro- and nanostructures.

\section{NONLOCAL EULER-BERNOULLI BEAM MODEL}

Let us consider a beam of length $L$ along the axial coordinate $x$. The Euler-Bernoulli beam theory considers the following displacement field:

$$
u_{1}=u(x, t)-y \frac{\partial v}{\partial x}, \quad u_{2}=v(x, t), \quad u_{3}=0,
$$

where $u$ and $v$ are, respectively, the axial and transverse displacements of the point $x$ on the middle plane (i.e., $y=0$ ) of the beam.

From this displacement field, the only nonvanishing strain is

$$
\varepsilon_{x x}=\frac{\partial u}{\partial x}-y \frac{\partial^{2} v}{\partial x^{2}}
$$

If the rotational inertia is neglected, the equations of motion of the beam is given by

$$
\frac{\partial N}{\partial x}+f(x, t)=\rho A \frac{\partial^{2} u}{\partial t^{2}}
$$

$$
\frac{\partial^{2} M}{\partial x^{2}}+p(x, t)=\rho A \frac{\partial^{2} v}{\partial t^{2}}
$$

where $\rho$ is the density of the material, $A$ is the cross-sectional area of the beam, $p$ is the vertical distributed transverse force along axis $x, f$ is the horizontal distributed transverse force along axis $x, N$ is the axial force, $S$ is the shear force, $M$ is the resultant bending moment, and $I$ is the moment of inertia, defined respectively as follows:

$$
\begin{aligned}
& N=\int_{A} \sigma_{x x} d A, \quad S=\int_{A} \sigma_{x y} d A, \\
& M=-\int_{A} \sigma_{x x} y d A, \quad I=\int_{A} y^{2} d A .
\end{aligned}
$$

The nonlocal expressions for the axial force, the bending moment, and the shear force can be determined from the constitutive equations as a function of the horizontal and vertical displacements and the forces distributed along the beam [Reddy (2007) and Reddy and Pang (2008)],

$$
\begin{aligned}
& N(x)=E A \frac{\partial u}{\partial x}+\left(a e_{0}\right)^{2}\left(\rho A \frac{\partial^{3} u}{\partial x \partial t^{2}}-\frac{\partial f}{\partial x}\right), \\
& M(x)=E I \frac{\partial^{2} v}{\partial x^{2}}-\left(a e_{0}\right)^{2}\left(p-\rho A \frac{\partial^{2} v}{\partial t^{2}}\right), \\
& S(x)=-E I \frac{\partial^{3} v}{\partial x^{3}}-\left(a e_{0}\right)^{2}\left(\frac{\partial p}{\partial x}-\rho A \frac{\partial^{3} v}{\partial x \partial t^{2}}\right) .
\end{aligned}
$$

Finally, the equations for the horizontal and vertical displacements for the nonlocal beam could be written as [Reddy (2007) and Reddy and Pang (2008)]

$$
\begin{aligned}
& E A \frac{\partial^{2} u}{\partial x^{2}}+f-\left(a e_{0}\right)^{2} \frac{\partial^{2} f}{\partial x^{2}}=\rho A \frac{\partial^{2} u}{\partial t^{2}}, \\
& E I \frac{\partial^{4} v}{\partial x^{4}}+\rho A\left(\frac{\partial^{2} v}{\partial t^{2}}-\left(a e_{0}\right)^{2} \frac{\partial^{4} v}{\partial x^{2} \partial t^{2}}\right)=p-\left(a e_{0}\right)^{2} \frac{\partial^{2} p}{\partial x^{2}} .
\end{aligned}
$$

In this work, transverse free vibrations are investigated. In order to solve the problem, only Eq. (12) must be analysed, by making the external load equal to zero,

$$
E I \frac{\partial^{4} v}{\partial x^{4}}+\rho A\left(\frac{\partial^{2} v}{\partial t^{2}}-\left(a e_{0}\right)^{2} \frac{\partial^{4} v}{\partial x^{2} \partial t^{2}}\right)=0 .
$$

The above equation can be solved by using the classical separation-of-variables method as

$$
v(x, t)=V(x) e^{i \omega t},
$$

where $\omega$ is the natural frequency of vibrations.

Substituting Eq. (14) in Eq. (13) and using the dimensionless variables and constants given by

$$
\xi=x / L, \quad h=\frac{a e_{0}}{L}, \quad \lambda^{4}=\frac{\rho A L^{4}}{E I} \omega^{2}, \quad \bar{V}=V / L,
$$

we get the spatial equation as 

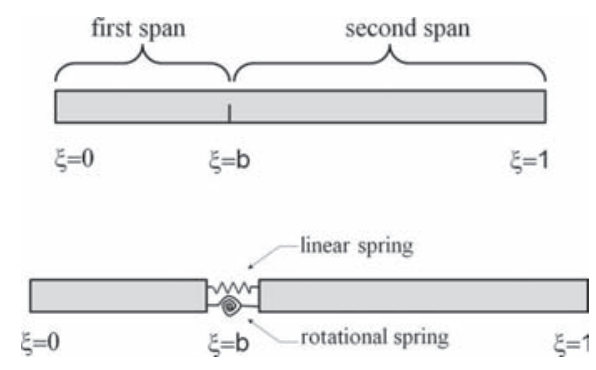

FIG. 1. Model of the cracked beam.

$$
\bar{V}^{I V}+\lambda^{4}\left(h^{2} \bar{V}^{\prime \prime}-\bar{V}\right)=0
$$

where $(\cdot)^{\prime}$ represents the derivative with respect to $\xi$. The general solution for the above differential equation is

$$
\begin{aligned}
\bar{V}(\xi)= & A_{1} \sinh \left(\beta_{r} \xi\right)+A_{2} \cosh \left(\beta_{r} \xi\right)+A_{3} \sin \left(\beta_{e} \xi\right) \\
& +A_{4} \cos \left(\beta_{e} \xi\right)
\end{aligned}
$$

where

$$
\begin{aligned}
& \beta_{r}=\lambda^{2} h \sqrt{\frac{\sqrt{1+4 / h^{4} \lambda^{4}}-1}{2}}, \\
& \beta_{e}=\lambda^{2} h \sqrt{\frac{\sqrt{1+4 / h^{4} \lambda^{4}}+1}{2}} .
\end{aligned}
$$

Once the displacements are known [Eq. (17)], and using the definition for the variables [Eq. (15)], the bending slope $\theta(\xi)$, the dimensionless bending moment $\bar{M}(\xi)$, and dimensionless shear force $\bar{S}(\xi)$ can be calculated as follows:

$$
\begin{aligned}
& \theta(\xi)=\bar{V}^{\prime}(\xi), \\
& \bar{M}(\xi)=\frac{M(\xi) L}{E I}=\bar{V}^{\prime \prime}(\xi)+h^{2} \lambda^{4} \bar{V}(\xi), \\
& \bar{S}(\xi)=\frac{S(\xi) L^{2}}{E I}=\bar{V}^{\prime \prime \prime}(\xi)+h^{2} \lambda^{4} \bar{V}^{\prime}(\xi) .
\end{aligned}
$$

Note that Eqs. (21) and (22) are easily derived from Eqs. (10), and they differ from the ones given by the local bending theory.

The four constants $A_{i}$ of the solution [Eq. (17)] are determined by solving the system of four equations that result from applying the proper boundary conditions on the end supports as follows:

- Simply supported end,

$$
\bar{V}=0 \text {, }
$$

$$
\bar{V}^{\prime \prime}+h^{2} \lambda^{4} V=0 \quad(\bar{M}=0),
$$

- fixed end,

$$
\bar{V}=0 \text {, }
$$

$\bar{V}^{\prime}=0$,

- free end,

$$
\begin{aligned}
& \bar{V}^{\prime \prime}+h^{2} \lambda^{4} \bar{V}=0 \quad(\bar{M}=0), \\
& \bar{V}^{\prime \prime \prime}+h^{2} \lambda^{4} \bar{V}^{\prime}=0 \quad(\bar{S}=0) .
\end{aligned}
$$

\section{NONLOCAL EULER-BERNOULLI CRACKED-BEAM MODEL}

Let us now consider that the beam has an edge crack of length $a$ located at a distance $L^{*}\left(b=L^{*} / L\right)$ from the left end. To take into account the effect of the crack, we extend the method first proposed by Freund and Herrmann (1976) and

\begin{tabular}{|c|c|c|c|c|c|c|c|c|}
\hline & \multicolumn{4}{|c|}{$h=0.0$} & \multicolumn{4}{|c|}{$h=0.2$} \\
\hline$\Lambda$ & $K=0$ & $K=0.065$ & $K=0.35$ & $K=2$ & $K=0$ & $K=0.065$ & $K=0.35$ & $K=2$ \\
\hline 1 & 3.1416 & 3.0469 & 2.7496 & 2.0960 & 2.8908 & 2.8031 & 2.5233 & 1.9098 \\
\hline 2 & 6.2832 & 6.2832 & 6.2832 & 6.2832 & 4.9581 & 4.9581 & 4.9581 & 4.9581 \\
\hline 3 & 9.4248 & 9.1669 & 8.6129 & 8.0730 & 6.4520 & 6.2604 & 5.7891 & 5.3416 \\
\hline \multirow[t]{2}{*}{4} & 12.5664 & 12.5664 & 12.5664 & 12.5664 & 7.6407 & 7.6407 & 7.6407 & 7.6407 \\
\hline & \multicolumn{4}{|c|}{$h=0.4$} & \multicolumn{4}{|c|}{$h=0.6$} \\
\hline 1 & 2.4790 & 2.4032 & 2.1567 & 1.6195 & 2.1507 & 2.0846 & 1.8678 & 1.3971 \\
\hline 2 & 3.8204 & 3.8204 & 3.8204 & 3.8204 & 3.1815 & 3.1815 & 3.1815 & 3.1815 \\
\hline 3 & 4.7723 & 4.6284 & 4.2729 & 3.9563 & 3.9329 & 3.8141 & 3.5226 & 3.2709 \\
\hline 4 & 5.5509 & 5.5509 & 5.5509 & 5.5509 & 4.5566 & 4.5566 & 4.5566 & 4.5566 \\
\hline
\end{tabular}
further followed by many others [Adams et al. (1978), Narkis (1994), Fernandez-Saez et al. (1999), Fernandez-Saez and Navarro (2002), and Loya et al. (2006)]. Thus, the cracked-beam has been considered to be two beams connected by a rotational and a longitudinal elastic spring at the cracked section (Fig. 1). Both springs are introduced to consider the additional strain energy caused by the presence of the crack.

In effect, the strain energy $\mathcal{U}$ of the cracked nanobeam can be stated as

TABLE I. Values of the first four order frequency parameters for a simply supported beam with different nonlocal parameter $h$ and crack-severity $K$. Crack position $\xi=0.50$. 
TABLE II. Values of the first four order frequency parameters for a simply supported beam with different nonlocal parameter $h$ and crack-severity $K$. Crack position $\xi=0.25$.

\begin{tabular}{ccccccccc}
\hline \hline \multicolumn{3}{c}{$h=0.0$} \\
\hline$\Lambda$ & $K=0$ & $K=0.065$ & $K=0.35$ & $K=2$ & $K=0$ & $K=0.065$ & $K=0.35$ & $K=2$ \\
\hline 1 & 3.1416 & 3.0921 & 2.9071 & 2.3493 & 2.8908 & 2.8447 & 2.6645 & 2.1134 \\
2 & 6.2832 & 6.1028 & 5.6491 & 5.1047 & 4.9580 & 4.8101 & 4.4169 & 3.9906 \\
3 & 9.4248 & 9.3021 & 9.0767 & 8.9008 & 6.4520 & 6.3638 & 6.1924 & 6.0692 \\
4 & 12.5664 & 12.5664 & 12.5664 & 12.5664 & 7.6407 & 7.6407 & 7.6407 & 7.6407 \\
& & & & & & & \\
& \multicolumn{2}{c}{$h=0.4$} & & & & & \\
\hline 1 & 2.4790 & 2.4387 & 2.2730 & 1.7660 & 2.1507 & 2.1154 & 1.9661 & 1.5125 \\
2 & 3.8203 & 3.70467 & 3.3993 & 3.1123 & 3.1815 & 3.0849 & 2.8336 & 2.6147 \\
3 & 4.7723 & 4.7072 & 4.5859 & 4.5062 & 3.9329 & 3.8795 & 3.7816 & 3.7191 \\
4 & 5.5509 & 5.5509 & 5.5509 & 5.5509 & 4.5566 & 4.5566 & 4.5566 & 4.5566 \\
\hline \hline
\end{tabular}

$$
\mathcal{U}=\frac{1}{2} \int_{0}^{L} d x \int_{A} \sigma_{x x}\left(\frac{\partial u}{\partial x}-y \frac{\partial^{2} v}{\partial x^{2}}\right) d A+\Delta \mathcal{U}_{c}
$$

with $\Delta \mathcal{U}_{c}$ being the increment of strain energy corresponding to the crack. This expression could be rewritten easily, using the definitions in Eq. (9) in terms of the axial force and the bending moment,

$$
\mathcal{U}=\frac{1}{2} \int_{0}^{L}\left(N \frac{\partial u}{\partial x}+M \frac{\partial^{2} v}{\partial x^{2}}\right) d x+\Delta \mathcal{U}_{c}
$$
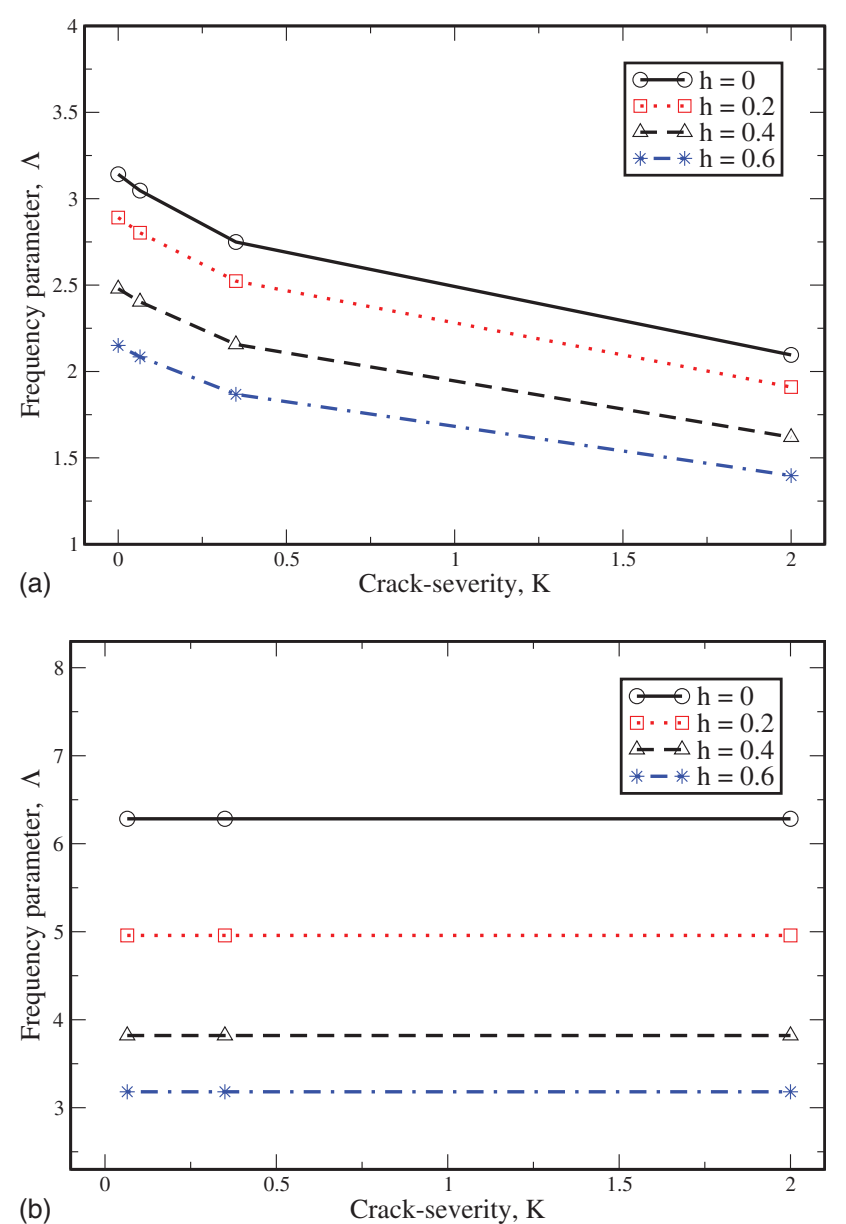

FIG. 2. (Color online) First four frequency parameters for a simply supported beam with crack position $\xi=0.50$.
In view of the functional form of the strain energy of the intact beam, we can write the increment due to the presence of an edge crack as follows:

$$
\begin{aligned}
\Delta \mathcal{U}_{c}= & \frac{1}{2} k_{M M} M\left(L^{*}, t\right) \frac{\partial^{2} v}{\partial x^{2}}+\frac{1}{2} k_{N N} N\left(L^{*}, t\right) \frac{\partial u}{\partial x} \\
& +\frac{1}{2} k_{M N} M\left(L^{*}, t\right) \frac{\partial u}{\partial x}+\frac{1}{2} k_{N M} N\left(L^{*}, t\right) \frac{\partial^{2} v}{\partial x^{2}}
\end{aligned}
$$

where $k_{N N}, k_{M M}, k_{M N}$, and $k_{N M}$ are the flexibility constants.
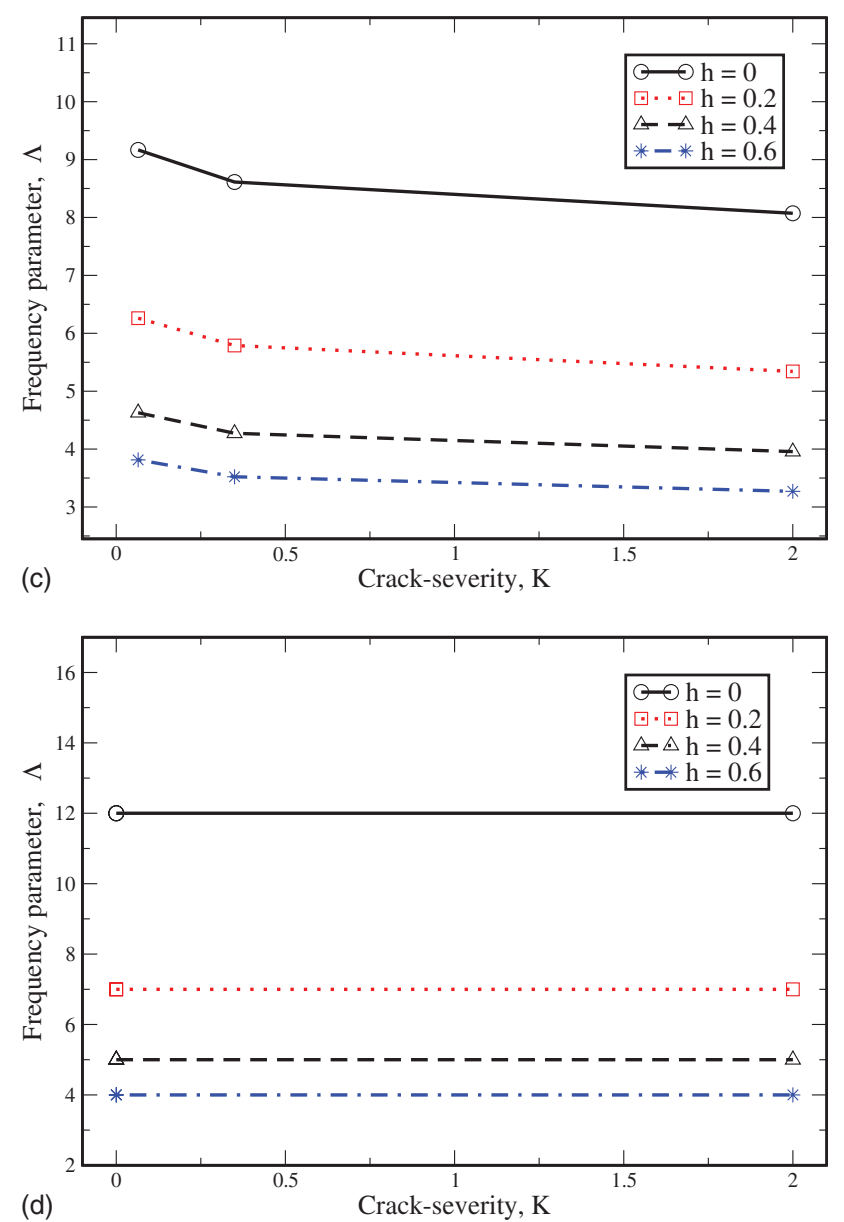

(d) 

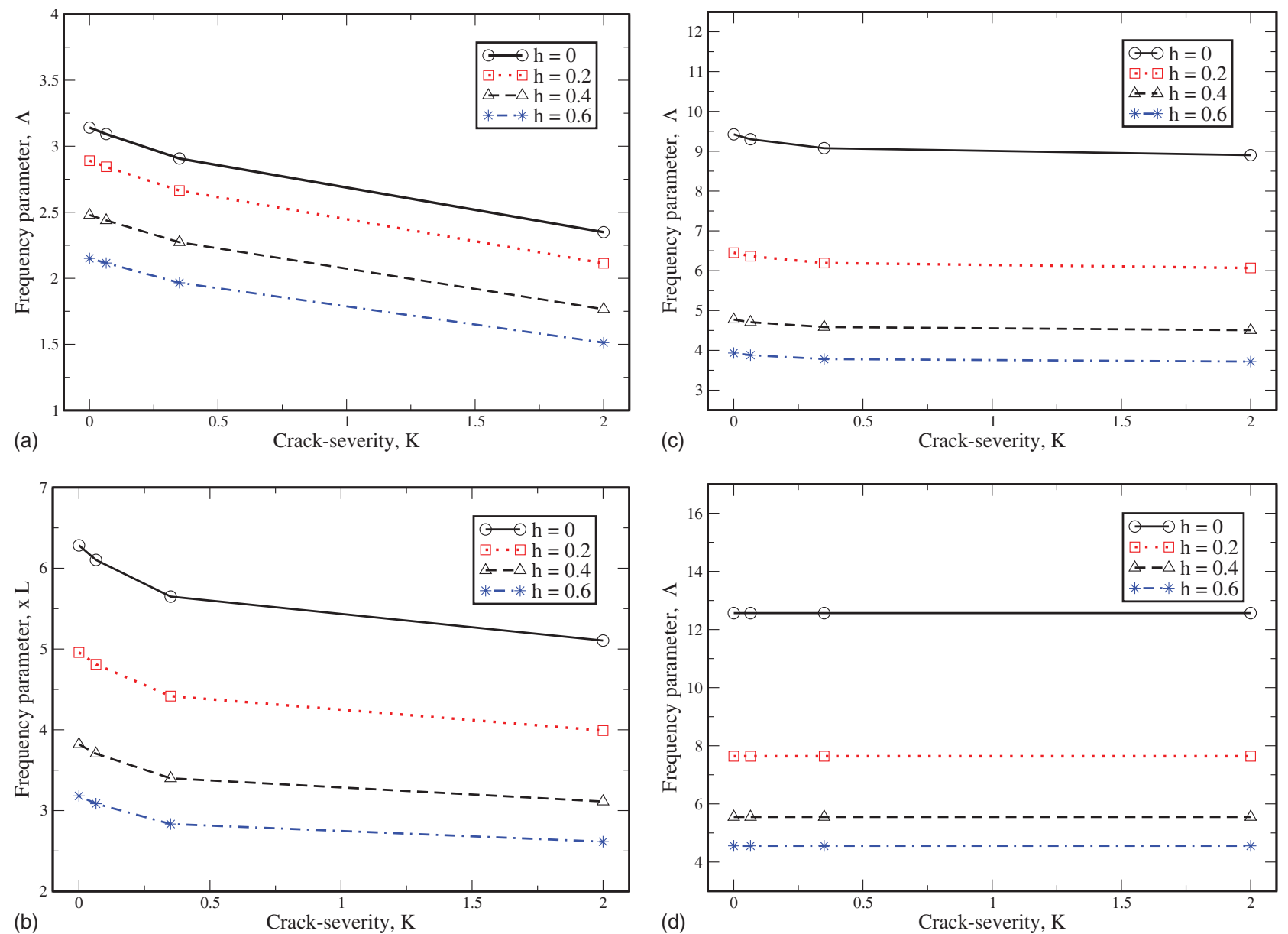

FIG. 3. (Color online) First four frequency parameters for a simply supported beam with crack position $\xi=0.25$.

The last two terms are considered to take into account the coupled effects between the axial force and bending moment. The increment of strain energy could be written as

$$
\Delta \mathcal{U}_{c}=\frac{1}{2} M \Delta \theta+\frac{1}{2} N \Delta u,
$$

$\Delta \theta$ being the angle rotated by the rotational spring and $\Delta u$ the relative horizontal displacement at the edge crack section. Comparing Eqs. (31) and (32) the following relations are established:

$$
\begin{aligned}
& \Delta \theta=k_{M M} \frac{\partial^{2} v}{\partial x^{2}}+k_{M N} \frac{\partial u}{\partial x}, \\
& \Delta u=k_{N N} \frac{\partial u}{\partial x}+k_{N M} \frac{\partial^{2} v}{\partial x^{2}} .
\end{aligned}
$$

As mentioned above, in this work the transverse free vibrations are investigated, and hence no longitudinal displacement is considered. In addition, the crossover flexibility constants $\left(k_{M N}\right.$ and $\left.k_{N M}\right)$ are assumed to be small enough, as in the case of local elasticity, to consider only the one related to the bending moment $\left(k_{M M}\right)$. The slope increment $\Delta \theta$ $=k_{M M} \partial^{2} v / \partial x^{2}$ in the cracked section is then expressed using dimensionless variables as

$$
\Delta \theta=\left.\frac{k_{M M}}{L} \frac{\partial^{2} \bar{V}(\xi)}{\partial \xi^{2}}\right|_{\xi=b}=\left.K \frac{\partial^{2} \bar{V}(\xi)}{\partial \xi^{2}}\right|_{\xi=b},
$$

where $K=k_{M M} / L$. In the case of local cracked beams, the additional strain energy due to the crack $\Delta \mathcal{U}_{c}$ can be calculated from the fracture mechanics theory [Irwin (1960)]. In the case of nanobeams, $\Delta \mathcal{U}_{c}$ must be obtained from either $a b$ initio studies or molecular dynamics calculations.

Once defined how the edge crack is modeled, we can proceed to solve the free transverse vibrations of cracked nanobeams. The spatial solution for the motion in the vertical direction for each of the two segments can be expressed in a way similar to Eq. (16),

$$
\begin{aligned}
& \bar{V}_{1}^{I V}+\Lambda^{4}\left(h^{2} \bar{V}_{1}^{\prime \prime}-\bar{V}_{1}\right)=0, \quad 0 \leqslant \xi \leqslant b, \\
& \bar{V}_{2}^{I V}+\Lambda^{4}\left(h^{2} \bar{V}_{2}^{\prime \prime}-\bar{V}_{2}\right)=0, \quad b \leqslant \xi \leqslant 1,
\end{aligned}
$$

with $\Lambda$ being the frequency parameter of the cracked beam, which could be related to its natural frequency $\omega_{c}$ by

$$
\Lambda^{4}=\frac{\rho A L^{4}}{E I} \omega_{c}^{2} .
$$

Thus, the general solution for the above differential equations Eq. (36) is 
TABLE III. Values of the first four order frequency parameters for a clamped-clamped beam with different nonlocal parameter $h$ and crack-severity $K$. Crack position $\xi=0.50$.

\begin{tabular}{lrrrrrrrr}
\hline \hline \multicolumn{9}{c}{$h=0.0$} \\
\hline$\Lambda$ & $K=0$ & $K=0.065$ & $K=0.35$ & $K=2$ & $K=0$ & $K=0.065$ & $K=0.35$ & $K=2$ \\
\hline 1 & 4.7300 & 4.6285 & 4.3566 & 3.9702 & 4.2766 & 4.1736 & 3.8855 & 3.4764 \\
2 & 7.8532 & 7.8532 & 7.8532 & 7.8532 & 6.0352 & 6.0352 & 6.0352 & 6.0352 \\
3 & 10.9956 & 10.6976 & 10.1028 & 9.5833 & 7.3840 & 7.1421 & 6.6089 & 6.2045 \\
4 & 14.1372 & 14.1372 & 14.1372 & 14.1372 & 8.4624 & 8.4624 & 8.4624 & 8.4624 \\
& & & & & & & \\
& & & & & & & & \\
\hline 1 & 3.5923 & 3.4947 & 3.2141 & 2.63306 & 3.0837 & 2.9952 & 2.7391 & 2.3949 \\
2 & 4.5978 & 4.5978 & 4.5978 & 4.5978 & 3.8165 & 3.8165 & 3.8165 & 3.8165 \\
3 & 5.4738 & 5.2993 & 4.9302 & 4.6170 & 4.5231 & 4.3831 & 4.0939 & 3.9116 \\
4 & 6.1504 & 6.1504 & 6.1504 & 6.1504 & 5.0505 & 5.0505 & 5.0505 & 5.0505 \\
\hline \hline
\end{tabular}

$$
\begin{aligned}
\bar{V}_{1}(\xi)= & A_{1} \sinh \left(\beta_{s} \xi\right)+A_{2} \cosh \left(\beta_{s} \xi\right)+A_{3} \sin \left(\beta_{f} \xi\right) \\
& +A_{4} \cos \left(\beta_{f} \xi\right), \quad 0 \leqslant \xi \leqslant b, \\
\bar{V}_{2}(\xi)= & A_{5} \sinh \left(\beta_{s} \xi\right)+A_{6} \cosh \left(\beta_{s} \xi\right)+A_{7} \sin \left(\beta_{f} \xi\right) \\
& +A_{8} \cos \left(\beta_{f} \xi\right), \quad b \leqslant \xi \leqslant 1,
\end{aligned}
$$

with $\beta_{s}$ and $\beta_{f}$ similar to Eqs. (18) and (19),

$$
\begin{aligned}
& \beta_{s}=\Lambda^{2} h \sqrt{\frac{\sqrt{1+4 / h^{4} \Lambda^{4}}-1}{2}}, \\
& \beta_{f}=\Lambda^{2} h \sqrt{\frac{\sqrt{1+4 / h^{4} \Lambda^{4}}+1}{2}} .
\end{aligned}
$$

The above two equations [Eqs. (38)], which present eight unknown constants, must be solved with the specified boundary conditions and the following compatibility conditions at the cracked section:

- Continuity of the vertical displacement,

$$
\bar{V}_{2}(b)=\bar{V}_{1}(b) \text {. }
$$

- Jump in bending slope,

$$
\Delta \theta=\bar{V}_{2}^{\prime}(b)-\bar{V}_{1}^{\prime}(b)=K \bar{V}_{1}^{\prime \prime}(b) .
$$

- Continuity of the bending moment,

$$
\bar{V}_{2}^{\prime \prime}(b)+h^{2} \Lambda^{4} \bar{V}_{2}(b)=\bar{V}_{1}^{\prime \prime}(b)+h^{2} \Lambda^{4} \bar{V}_{1}(b) .
$$

- Continuity of the shear force,

$$
\bar{V}_{2}^{\prime \prime \prime}(b)+h^{2} \Lambda^{4} \bar{V}_{2}^{\prime}(b)=\bar{V}_{1}^{\prime \prime \prime}(b)+h^{2} \Lambda^{4} \bar{V}_{1}^{\prime}(b) .
$$

Instead of eight unknown factors, the expressions for displacements [Eqs. (38)] may be written in a more convenient form in terms of just four constants: the displacement $V_{0}$, bending slope $\theta_{0}$, bending moment $M_{0}$, and shear force $S_{0}$ at $\xi=0$,

\begin{tabular}{|c|c|c|c|c|c|c|c|c|}
\hline \multirow[b]{2}{*}{$\Lambda$} & \multicolumn{4}{|c|}{$h=0.0$} & \multicolumn{4}{|c|}{$h=0.2$} \\
\hline & $K=0$ & $K=0.065$ & $K=0.35$ & $K=2$ & $K=0$ & $K=0.065$ & $K=0.35$ & $K=2$ \\
\hline 1 & 4.7300 & 4.7273 & 4.7194 & 4.7068 & 4.2766 & 4.2752 & 4.2705 & 4.2595 \\
\hline 2 & 7.8532 & 7.6991 & 7.3175 & 6.8770 & 6.0352 & 5.9062 & 5.5040 & 5.0234 \\
\hline 3 & 10.9956 & 10.7787 & 10.4067 & 10.1452 & 7.3840 & 7.1962 & 6.8797 & 6.7231 \\
\hline \multirow[t]{2}{*}{4} & 14.1372 & 14.0911 & 14.0159 & 13.9634 & 8.4490 & 8.3902 & 8.2780 & 8.2163 \\
\hline & \multicolumn{4}{|c|}{$h=0.04$} & \multicolumn{4}{|c|}{$h=0.6$} \\
\hline 1 & 3.5923 & 3.5920 & 3.5903 & 3.5810 & 3.0837 & 3.0836 & 3.0831 & 3.0690 \\
\hline 2 & 4.5978 & 4.4969 & 4.1539 & 3.7561 & 3.8165 & 3.7324 & 3.43941 & 3.1093 \\
\hline 3 & 5.4738 & 5.3242 & 5.0900 & 4.9941 & 4.5231 & 4.3965 & 4.20161 & 4.1256 \\
\hline 4 & 6.1504 & 6.0891 & 6.0081 & 5.9707 & 5.0505 & 4.9985 & 4.93391 & 4.9056 \\
\hline
\end{tabular}

$$
\bar{V}_{1}(\xi)=V_{0} g_{1}(\xi)+\theta_{0} g_{2}(\xi)+M_{0} g_{3}(\xi)+S_{0} g_{4}(\xi), \quad 0 \leqslant \xi \leqslant b,
$$

$$
\bar{V}_{2}(\xi)=\bar{V}_{1}(\xi)+\Delta \theta g_{2}(\xi-b), \quad b \leqslant \xi \leqslant 1,
$$

where the functions $g_{i}(\xi)$ can be expressed as follows:

$$
g_{1}(\xi)=\cosh \left(\xi \beta_{s}\right)+\frac{\left[\cos \left(\xi \beta_{f}\right)-\cosh \left(\xi \beta_{s}\right)\right]\left(h^{2} \Lambda^{4}+\beta_{s}^{2}\right)}{\beta_{f}^{2}+\beta_{s}^{2}}
$$

TABLE IV. Values of the first four order frequency parameters for a clamped-clamped beam with different nonlocal parameter $h$ and crack-severity $K$. Crack position $\xi=0.25$. 

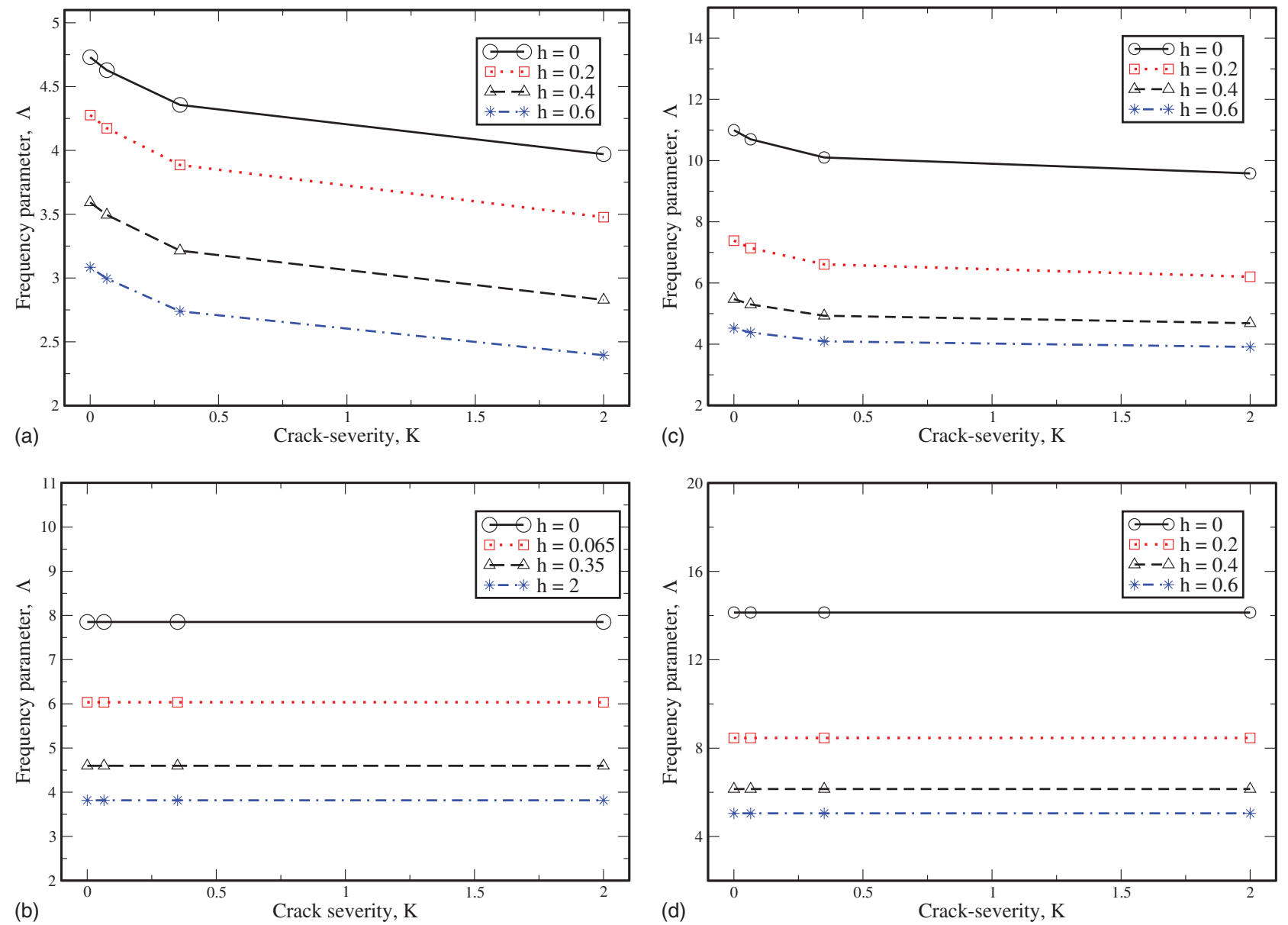

FIG. 4. (Color online) First four frequency parameters for a clamped-clamped beam with crack position $\xi=0.50$.

$$
\begin{aligned}
& g_{2}(\xi)=\frac{\sin \left(\xi \beta_{f}\right)}{\beta_{f}} \\
& -\frac{\left(-h^{2} \Lambda^{4} \beta_{f}+\beta_{f}^{3}\right)\left[-\sinh \left(\xi \beta_{s}\right) \beta_{f}+\sin \left(\xi \beta_{f}\right) \beta_{s}\right]}{\beta_{f}^{2} \beta_{s}\left(\beta_{f}^{2}+\beta_{s}^{2}\right)}, \\
& g_{3}(\xi)=-\frac{\cos \left(\xi \beta_{f}\right)-\cosh \left(\xi \beta_{s}\right)}{\beta_{f}^{2}+\beta_{s}^{2}}, \\
& g_{4}(\xi)=\frac{-\sinh \left(\xi \beta_{s}\right) \beta_{f}+\sin \left(\xi \beta_{f}\right) \beta_{s}}{\beta_{f} \beta_{s}\left(\beta_{f}^{2}+\beta_{s}^{2}\right)} .
\end{aligned}
$$

the use of a $8 \times 8$ determinant; in addition, this technique is suitable to analyze the presence of multiple cracks in nanobeams, without increasing the difficulty of the problem and keeping the resolution by the $2 \times 2$ determinant.

The general solution expressed in this way [Eqs. (45) and (46)] satisfy the compatibly conditions at the cracked section [Eqs. (41)-(44)], and two of the constants are determined directly from the type of support at $\xi=0$. The other two unknown constants are determined by the system of two equations from the proper boundary conditions at $\xi=1$. In case of $n$ cracks, an extra equation similar to Eq. (46) must be added for every new segment, and regardless of the number of cracks, the problem can be solved by a $2 \times 2$ system of equations. This is a homogenous system, and to avoid the trivial solution it is necessary to impose the nullity of the determinant of the coefficients' matrix. The methodology used considerably simplifies the calculations, compared with

\section{RESULTS}

The results presented in this section correspond to the first four natural frequencies of a nonlocal Euler-Bernoulli beam for different positions for the edge crack (at the midspan, $\xi=0.5$, and at a quarter of the beam $\xi=0.25$ ), two different boundary conditions (simply supported and clampedclamped), and different crack-severities $K$. As mentioned above, the values of $K$ as a function of size of defect must be determined for nanobeams from molecular dynamics models; in this work values of $K=0$ (uncracked beam), and $K$ $=0.065,0.35$, and 2 were used. The nonlocal effect was introduced using the parameter $h$. Results were obtained for values of nonlocal parameter $h$ of 0 (local conventional case), 0.2, 0.4, and 0.6. A total of 24 cases were studied.

\section{A. Simply supported beam}

The changes of the first four eigenvalues $\Lambda_{i}$ for the simply supported beam with the crack-severity parameter $K$ for 

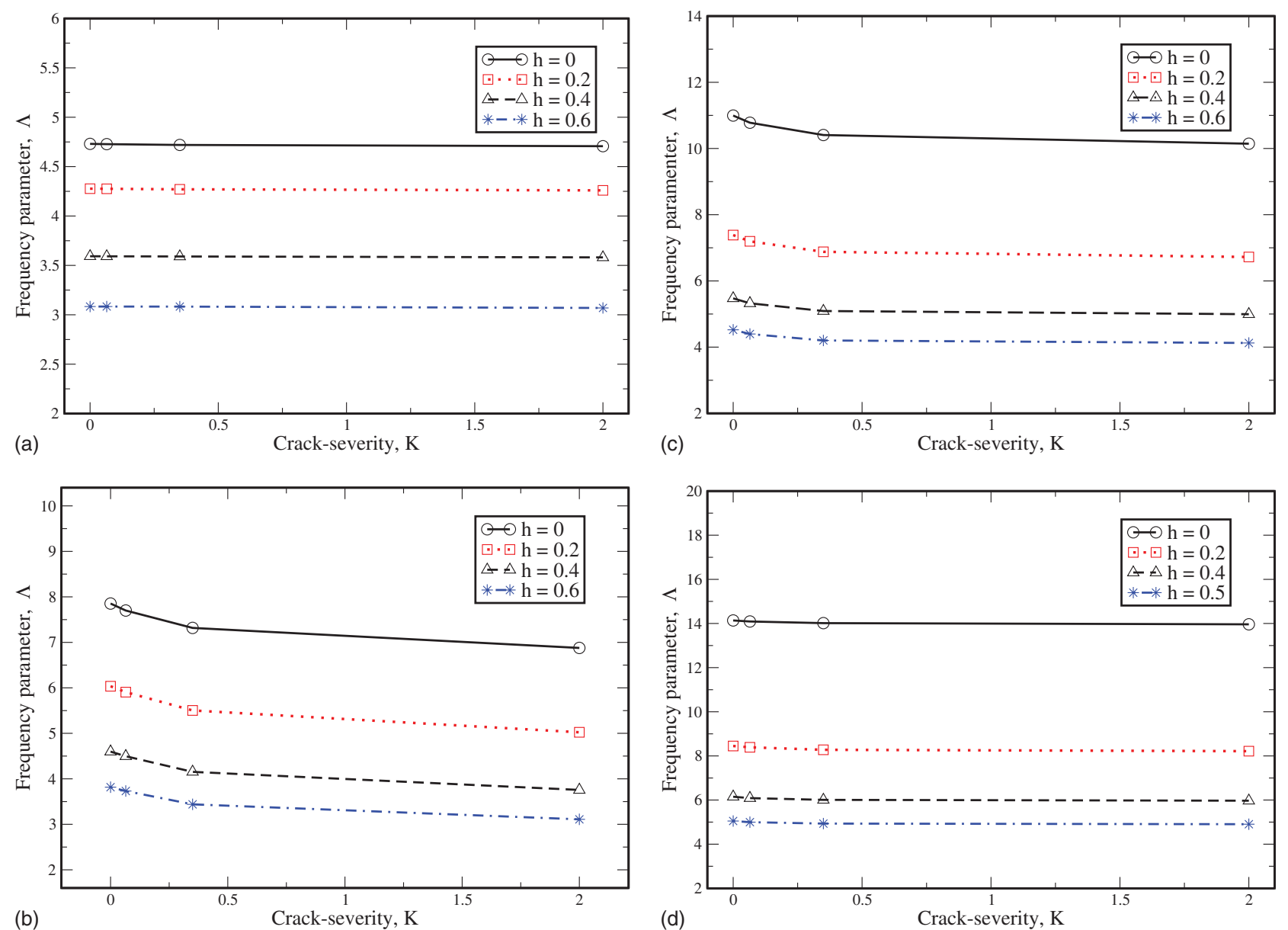

FIG. 5. (Color online) First four frequency parameters for a clamped-clamped beam with crack position $\xi=0.25$.

different values of $h$ are given in Table I in the case of an edge crack located at the midspan of the beam, and in Table II for a crack located at $\xi=0.25$.

In both cases, the first four natural frequencies decrease with the local parameter $h$. For the uncracked beam, the values found exactly reproduce the results given by $\mathrm{Lu}$ (2007) for different values of $h$. In local cracked beams, this trend is also observed; it is also found that as the crack considered lengthens, the beam stiffness decreases and so do the natural frequencies.

Due to the symmetry of the problem, the mid-span cracked beam has the second and fourth natural frequencies independent of crack-severity; this behavior was expected from the fact that the second derivative of vertical displacement is canceled in the cracked section. The same result was found for the beam with the crack position at $\xi=0.25$, but only for the fourth natural frequency.

In Figs. 2 and 3 it can be seen that the nonlocal effect has a significant influence on the dynamic properties of a cracked beam, in way similar to the first four order eigenvalues for any edge-crack length. The difference between the results found using local and nonlocal beam theory is greater in higher modes.

\section{B. Clamped-clamped beam}

The changes of the first four order eigenvalues $\Lambda_{i}$ for the clamped-clamped beam with the crack-severity parameter $K$ for different values of parameter $h$ are given for a crack located at $\xi=0.5$ and $\xi=0.25$ in Tables III and IV, respectively.

It was found that the variations of the natural frequencies with $K$ and $h$ for the clamped beam (see Figs. 4 and 5) have trends similar to those shown in Figs. 2 and 3 for the simply supported beam, since the clamped beam is stiffer than the simply supported beam for a pair of given nonlocal and crack parameters.

As in the simple-supported beam with $\xi=0.50$, the symmetry of the problem causes the second and fourth modal frequencies not to be crack dependent; the same nondependency is observed in the fourth mode for the quarterspan cracked beam.

\section{CONCLUSIONS}

This paper examines the natural frequencies of cracked nanobeams; the nonlocal elasticity theory was used to take into account the size effect of the structure analyzed. The cracked nanobeam was modeled using a rotational spring which promotes a discontinuity in the slope. This discontinuity is proportional to the crack severity. Two different boundary conditions were considered: simply supported and clamped-clamped.

The methodology used allows the simple determination of the first four natural frequencies and hence the possibility 
of analyzing the influence of the most important parameters that appear in these kinds of problems: crack severity, crack position, and nonlocal effect.

From these results presented and discussed, the main conclusions are as follows:

- The nonlocal parameter $h$ considerably affects the value of the natural frequencies, which decreases as $h$ increases.

- The influence of the nonlocal parameter is stronger at higher modes rather than at the lower ones.

- For the uncracked case, $K=0$, and taken into account the nonlocal effect, the results are compared with the work of Lu (2007) and completely coincide.

\section{ACKNOWLEDGMENTS}

This research was done with the financial support of the University Carlos III of Madrid and of the Comunidad Autónoma de Madrid under Projects CCG06-UC3M/DPI-0796 and CCG07-UC3M/DPI-3395.

${ }^{1}$ R. D. Adams, P. Cawley, C. J. Pye, and B. J. Stone, J. Mech. Eng. Sci. 20, 93 (1978).

${ }^{2}$ A. C. Eringen, Int. J. Eng. Sci. 10, 1 (1972).

${ }^{3}$ A. C. Eringen, J. Appl. Phys. 54, 4703 (1983).

${ }^{4}$ A. C. Eringen, Nonlocal Continuum Field Theories (Springer-Verlag, New
York, 2002).

${ }^{5}$ A. C. Eringen and D. G. B. Edelen, Int. J. Eng. Sci. 10, 233 (1972).

${ }^{6}$ J. Fernandez-Saez and C. Navarro, 2002, J. Sound Vib. 256, 17 (2002).

J. Fernandez-Saez, L. Rubio, and C. Navarro, J. Sound Vib. 225, 345 (1999).

${ }^{8}$ L. B. Freund and G. Herrmann, J. Appl. Mech. 76-APM-15, 112 (1976).

${ }^{9}$ H. Heireche, A. Tounsi, A. Benzair, M. Maachou, and E. Adda Bedia, Physica E (Amsterdam) 40, 2791 (2008).

${ }^{10}$ G. Irwin, Structural Mechanics (Pergamon, New York, 1960), pp. 557592.

${ }^{11}$ J. Loya, L. Rubio, and J. Fernandez-Saez, J. Sound Vib. 290, 640 (2006).

${ }^{12}$ P. Lu, J. Appl. Phys. 101, 073504 (2007).

${ }^{13}$ P. Lu, H. P. Lee, C. Lu, and P. Q. Zhang, J. Appl. Phys. 99, 073510 (2006).

${ }^{14}$ P. Lu, H. P. Lee, C. Lu, and P. Q. Zhang, Int. J. Solids Struct., 44, 5289 (2007).

${ }^{15}$ A. Luque, J. Aldazabal, J. M. Martinez-Esnaola, and J. Gil Sevillano, Fatigue Fract. Eng. Mater. Struct. 29, 615 (2006).

${ }^{16}$ Y. Narkis, J. Sound Vib. 172, 549 (1994).

${ }^{17}$ J. Peddieson, G. R. Buchanan, and R. P. McNitt, Int. J. Eng. Sci. 41, 305 (2003).

${ }^{18}$ J. N. Reddy, Int. J. Eng. Sci. 45, 288 (2007).

${ }^{19}$ J. N. Reddy and S. D. Pang, J. Appl. Phys. 103, 023511 (2008).

${ }^{20}$ L. Sudak, J. Appl. Phys. 94, 7281 (2003).

${ }^{21}$ C. M. Wang, Y. Y. Zhang, and X. Q. He, Nanotechnology 18, 105401 (2007).

${ }^{22}$ C. M. Wang, Y. Y. Zhang, S. S. Ramesh, and S. Kitipornchai, J. Phys. D 39, 3904 (2006).

${ }^{23}$ Q. Wang, J. Appl. Phys. 98, 124301 (2005).

${ }^{24}$ Q. Wang and K. Liew, Phys. Lett. A 363, 236 (2007).

${ }^{25}$ Q. Wang and V. K. Varadan, Smart Mater. Struct. 15, 659 (2006).

${ }^{26}$ M. T. Xu, Proc. R. Soc. London, Ser. A 462, 2977 (2006). 